\title{
System for interstitial photodynamic therapy with online dosimetry: first clinical experiences of prostate cancer
}

\author{
Johannes Swartling \\ SpectraCure $A B$ \\ Magistratsvägen 10 \\ SE-226 43 Lund, Sweden
}

\author{
Johan Axelsson \\ Lund University \\ Department of Physics \\ P.O. Box 118 \\ SE-221 00 Lund, Sweden
}

\section{Göran Ahlgren}

Malmö University Hospital

Department of Urology

SE-205 02 Malmö, Sweden

\section{Karl Mikael Kälkner}

Sten Nilsson

Karolinska University Hospital

Department of Oncology

Radiumhemmet

SE-171 76 Stockholm, Sweden

\author{
Sune Svanberg \\ Lund University \\ Department of Physics \\ P.O. Box 118 \\ SE-221 00 Lund, Sweden
}

\section{Katarina Svanberg}

Lund University Hospital

Department of Oncology

SE-221 85 Lund, Sweden

\author{
Stefan Andersson-Engels \\ Lund University \\ Department of Physics \\ P.O. Box 118 \\ SE-221 00 Lund, Sweden
}

\begin{abstract}
The first results from a clinical study for Temoporfinmediated photodynamic therapy (PDT) of low-grade (T1c) primary prostate cancer using online dosimetry are presented. Dosimetric feedback in real time was applied, for the first time to our knowledge, in interstitial photodynamic therapy. The dosimetry software IDOSE provided dose plans, including optical fiber positions and light doses based on 3-D tissue models generated from ultrasound images. Tissue optical property measurements were obtained using the same fibers used for light delivery. Measurements were taken before, during, and after the treatment session. On the basis of these real-time measured optical properties, the light-dose plan was recalculated. The aim of the treatment was to ablate the entire prostate while minimizing exposure to surrounding organs. The results indicate that online dosimetry based on real-time tissue optical property measurements enabled the light dose to be adapted and optimized. However, histopathological analysis of tissue biopsies taken six months post-PDT treatment showed there were still residual viable cancer cells present in the prostate tissue sections. The authors propose that the incomplete treatment of the prostate tissue could be due to a too low light threshold dose, which was set to $5 \mathrm{~J} / \mathrm{cm}^{2}$. $\odot 2010$ Society of Photo-Optical Instrumentation Engineers. [DOI: 10.1117/1.3495720]
\end{abstract}

Keywords: photodynamic therapy; biomedical optics; spectrometry; laser applications.

Paper 10185R received Apr. 9, 2010; revised manuscript received Aug. 19, 2010; accepted for publication Aug. 20, 2010; published online Oct. 27, 2010.

\section{Introduction}

Photodynamic therapy (PDT) has long been used for treatment of superficial lesions, for instance, of the skin. Over the years, much research in PDT has been devoted to enhancing the modality for treatment of larger and more deeply located lesions. The introduction of interstitial light delivery in PDT has provided the potential for treatment of such lesions. Inter-

Address all correspondence to: Stefan Andersson-Engels, Lund University, Department of Physics, P.O. Box 118, SE-221 00 Lund, Sweden; Tel: 46-46-22-3121; Fax: 46-46-222-42-50; E-mail: stefan.andersson-engels@fysik.Ith.se stitial PDT of internal cancer tumors is now investigated in a few relatively large clinical studies. ${ }^{1-5}$ Historically, the main difficulties of this treatment approach have been related to several issues. The fundamental issue is the limited penetration depth of light. Another difficulty in an interstitial setting is access and guidance of the light to the tumor. The third issue is to find the right dose parameters both in terms of light and photosensitizer while taking local tissue oxygenation into account. The first two issues are solved by inserting multiple

1083-3668/2010/15(5)/058003/9/\$25.00 @ 2010 SPIE 
optical fibers into the target tissue by means of image guidance technology, often adapted from procedures in interventional radiology and brachytherapy. ${ }^{6,7}$

The latter issue of finding the most optimal light and photosensitizer dose has been an area of much research in PDT therapeutics. Efforts in interstitial PDT have thus far often been hampered by large variability in the treatment outcome. It has been suggested that this is due to poor control of local photosensitizer concentration, ${ }^{8,9}$ the local light dose in the tissue, ${ }^{10,11}$ and unknown tissue oxygen concentration. ${ }^{12-14}$ These factors may lead to large variability in the locally deposited dose of singlet oxygen and other reactive oxidative species.

In order to solve some of the issues related to variability in treatment outcome in interstitial PDT, a number of groups have developed approaches to improve interstitial PDT dosimetry. An active clinical program for PDT of prostate cancer using the photosensitizer WST09 $\left(\right.$ Tookad $\left.^{\circledR}\right)$ has been pursued at University Health Network, Toronto. ${ }^{2,3}$ Cylindrical diffuser fibers for light delivery and spherical diffusers for monitoring were utilized ${ }^{6}$ in a clinical trial, including patients with recurrent prostate cancer after external beam radiation. The dosimetric consideration was to target the whole prostate gland with a fluence dose above a certain threshold dose. Post-treatment dose calculations were accomplished with a scheme based on the finite element method where the target volume was rendered from magnetic resonance (MR) images. ${ }^{15}$

Promising results have also been reported using motexafin lutetium (Lutex) in the management of prostate cancer. ${ }^{4,5} \mathrm{~A}$ number of techniques were employed during one clinical trial involving measurements of optical properties, ${ }^{16}$ fluorescence spectroscopy of photosensitizer, ${ }^{9}$ and optical assessment of tissue oxygenation. ${ }^{17}$ The measurements were performed by translatable spherical diffuser fibers allowing the light fluence rate to be measured directly. The intent was to target the entire gland, which was accomplished through relevant spacing between the diffusers. In addition, dose-planning algorithms have been reported by this group both for optimizing light delivery parameters ${ }^{18}$ and to tackle the problem of heterogeneous tissue types within the target tissue. ${ }^{19}$

Our group has been working to develop a system for interactively controlling the treatment to achieve a treatment optimally adapted for each individual treatment. The development of our system for interstitial PDT with online dosimetry has been described previously, e.g., Refs. 20 and 21. The algorithms and computer software that control the dosimetry have also been described. ${ }^{22,23}$ A unique feature of our system is that the same optical fibers are used for light delivery and measurements, a functionality that is provided by an optical switch.

Prostate cancer was chosen as the first cancer type for this interstitial PDT system because of the medical interest in novel treatment methods for this indication and because the prostate is relatively easy to access. Fiber insertion can be similar to radioactive seed insertion in brachytherapy. As mentioned previously, interstitial PDT is being explored actively by several groups as a potential novel treatment of localized prostate cancer. The current curative treatment options are surgery, external beam radiation therapy, or brachytherapy. PDT potentially offers several advantages: minimally invasive treatment, few side effects, rapid recovery, repeatable treat- ment if needed, and does not exclude other treatment options.

The purpose of this paper is to provide a description of an interstitial PDT system based on computer-controlled online feedback from a technical and dosimetry perspective, and we report our initial clinical experiences. To our knowledge, our system is the first to clinically incorporate online dosimetry by which monitoring is performed before and during treatment with immediate feedback to optimize the light dose. The clinical study is ongoing, and more comprehensive clinical results will be reported in future publications.

\section{Materials and Methods}

\subsection{Instrumentation}

The system, SpectraCure P18 with IDOSE $^{\circledR}$ (Interactive Dosimetry by Sequential Evaluation), is functionally similar to previously described instruments. ${ }^{20,21}$ However, for this study, it was scaled up to accomodate up to 18 optical fibers for light delivery and monitoring. The system can operate in either of two modes: light-delivery mode or monitoring mode. All optical fibers are used both for light delivery and monitoring of optical properties. Switching between the two modes is accomplished by means of an internal optomechanical fiber switch. Eighteen individually controlled diode lasers with a laser power of $250 \mathrm{~mW}$ and operating at a wavelength of $652 \mathrm{~nm}$ are used as light sources. The output power from each bare-end fiber is calibrated to $150 \mathrm{~mW}$ prior to each treatment session. Bare-end fibers are used to ensure welldefined measurement points. In monitoring mode, the system alternates between three different monitoring light sources: a laser diode of the same type as used for treatment, a blue light-emitting diode (LED) centered at $410 \mathrm{~nm}$, and a twin near-infrared (NIR) LED operating in the range of $750-850 \mathrm{~nm}$. While in monitoring mode, for each cluster of seven fibers, one fiber is emitting light while the other six surrounding fibers are used to collect scattered light. The detecting fibers are then coupled, through the optical switch, to individual spectrometers that operate in the range of 640-880 $\mathrm{nm}$. The monitoring cycle is completed when all 18 fibers have emitted light once, meaning that a full monitoring sequence results in $18 \times 6$ measurements for each of the three monitoring light sources. With this setup, it is possible to monitor the optical properties of the tissue at the treatment wavelength, the fluorescence of the photosensitizer excited at 410 and $652 \mathrm{~nm}$, and the oxygen saturation through NIR spectroscopy. The system also supports the use of up to six additional verification fibers, which are only used to monitor the light fluence at critical positions during treatment light delivery. The system is CE approved as a class IIb medical device for use with Foscan ${ }^{\circledR}$ for prostate cancer.

\subsection{Dosimetry}

A more detailed description of the dosimetry platform, IDOSE $^{\circledR}$, is found in Refs. 22 and 23. Briefly, a 3-D model of the prostate and surrounding tissues is generated from transrectal ultrasound (TRUS) images. The software calculates optimum fiber positions and presents the positions to the user. When the optical fibers are in place, a first monitoring sequence is performed. The optical properties are evaluated by assuming that the tissue is locally homogeneous around each subcluster of seven optical fibers (one emitter plus six receiv- 
Table 1 Tabulated optical properties for the intralipid phantoms as evaluated using TOF spectroscopy.

\begin{tabular}{lllllllll}
\hline Phantom & A & B & C & D & E & F & G & H \\
\hline$\mu_{a}\left(\mathrm{~cm}^{-1}\right)$ & 0.58 & 0.64 & 0.71 & 0.44 & 0.42 & 0.54 & 0.67 & 0.63 \\
$\mu_{s}^{\prime}\left(\mathrm{cm}^{-1}\right)$ & 8 & 7.7 & 7.7 & 7.4 & 10.3 & 9.2 & 7.9 & 10.7 \\
$\mu_{\text {eff }}\left(\mathrm{cm}^{-1}\right)$ & 3.86 & 4.0 & 4.23 & 3.22 & 3.68 & 3.97 & 4.15 & 4.63 \\
\hline
\end{tabular}

ers). A simple fit, based on the diffusion approximation, recovers the effective attenuation coefficient for each cluster. The effective attenuation coefficients are used to compute a dose plan that is presented to the user, who then has to approve the start of the treatment. At specific intervals, the light delivery is interrupted for monitoring sequences. In each monitoring sequence, the effective attenuation coefficients are evaluated and the dose plan is updated if significant changes occur to the optical properties that would perturb the delivered light dose. In the current dose planning algorithm, based on Cimmino's method, the aim is to deliver a minimum threshold light dose to all parts of the target volume while attempting to minimize exposure to the surrounding organs outside the target tissue. The light propagation is determined by the optical properties; hence, the basis for the dosimetry protocol is the measurement of these properties. In this study, the photosensitizer fluorescence and oxygen saturation data were not used in the computation of the dose plan because no dosimetry model to incorporate this information is yet available. Data are instead collected to serve as the basis for future development. Relying on the light fluence dose only and not taking the photosensitizer nor oxygenation into account is a simplification to the PDT dosimetry problem. Despite this simplification, it is a relevant first approach because the light fluence dose distribution is the only parameter that can be controlled during treatment by adjusting the light delivery time.

\subsection{Threshold Light Dose}

The threshold light dose was set to $5 \mathrm{~J} / \mathrm{cm}^{2}$ (fluence). This was based on estimates of the lesion size of necrosis determined by MR imaging (MRI) in the Moore et al. study, ${ }^{24,25}$ and data of optical properties of prostate tissue taken from a previous study. ${ }^{26}$ With a lesion radius of $7 \mathrm{~mm}$ and assuming homogeneous optical properties of $\mu_{\mathrm{a}}=0.6 \mathrm{~cm}^{-1}$ and $\mu_{\mathrm{s}}^{\prime}=10 \mathrm{~cm}^{-1}$, this yields an estimated threshold dose for tissue necrosis of $\sim 8.5 \mathrm{~J} / \mathrm{cm}^{2}$. This estimate was further verified by comparing to the EMEA-approved recommended light dose for superficial illumination, $20 \mathrm{~J} / \mathrm{cm}^{2}$. Assuming optical properties $\mu_{\mathrm{a}}=1 \mathrm{~cm}^{-1}$ and $\mu_{\mathrm{s}}^{\prime}=10 \mathrm{~cm}^{-1}$ and a depth of necrosis of $5 \mathrm{~mm}$, this yields a threshold dose of $6-10 \mathrm{~J} / \mathrm{cm}^{2}$ (fluence), which is in the same range as estimated from the prostate study. The threshold dose for this clinical study was conservatively set to $5 \mathrm{~J} / \mathrm{cm}^{2}$ as precaution to avoid damage to organs at risk because this was the first time IDOSE was used clinically.

\subsection{Tissue Phantom Experiments}

The capability of the instrument to assess optical properties interstitially was tested within an optical phantom study preclinically. Eight different optical phantoms composed of varying concentrations of Intralipid ${ }^{\odot}$ (Fresenius Kabi, Uppsala, Sweden), ink (Pelican Fount, Feusisberg, Switzerland), and water were made to mimic different scattering and absorption properties. Absorption and scattering coefficients, chosen to correspond to a broad range of relevant prostate optical properties, ${ }^{16,25,26}$ were evaluated separately using a time-offlight (TOF) spectroscopy instrument as described in Ref. 26. Briefly, this TOF instrument operates by sending a short laser pulse into the medium. The diffusely scattered light is detected, using time-correlated single-photon counting electronics, at some distance where the laser pulse is temporally dispersed. On the basis of the temporal dispersion, the absorption and scattering coefficients can be deduced. The optical properties are stated in Table 1. The fibers were positioned, using a fiber grid, into the liquid phantoms, and a complete treatment sequence was executed for each phantom.

\subsection{Clinical Study}

The clinical study was performed at Malmö University Hospital, Sweden, and Karolinska University Hospital, Stockholm, Sweden. The inclusion criteria for patients into this clinical study included histologically proven, untreated, organ-confined prostate cancer (stage T1c, Gleason score $<7$, PSA $<10 \mathrm{ng} / \mathrm{ml}$ ). This nonrandomized clinical study, approved by the Swedish Medical Products Agency, is currently still recruiting patients, and each patient has a follow-up time of 12 months. Patients were sensitized with Foscan (Biolitec Pharma) according to the European Medicines Agency (EMEA)-approved protocol for head and neck cancer: $0.15 \mathrm{mg} / \mathrm{kg}$ given intravenously, and the drug-light interval was four days. Patients were anesthetized either by general of epidural anaesthesia, placed in the lithotomy position, and TRUS images were acquired as transverse slices $5 \mathrm{~mm}$ apart aided by a mechanical stepper on which the ultrasound transducer was mounted (B-K Medical, Herlev, Denmark). Tissue contouring was performed by the urologist, and the system then computed the fiber positions following a threedimensional rendering of the target volume. Eighteen-gauge needles were inserted transperineally under TRUS guidance, aided by a mechanical grid template with a coordinate system calibrated to the ultrasound scanner. The actual coordinate positions, which could differ slightly from those recommended by the system, were reported back and entered into 


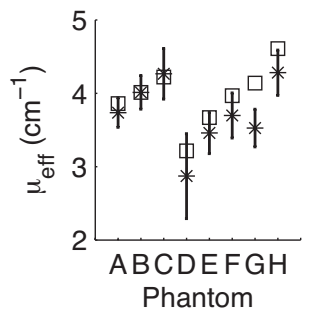

(a)

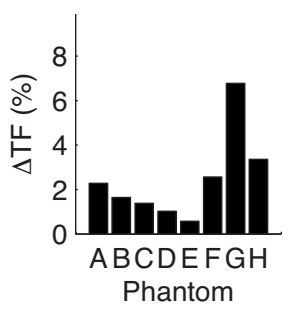

(b)
Fig. 1 (a) Evaluated effective attenuation coefficient using IDOSE (white) and TOF (black) for different tissue phantoms. The mean of the evaluated $\mu_{\text {eff }}$ for all 18 fibers are shown, and the error-bars indicate \pm 1 standard deviation. (b) The relative difference in treatment fraction, defined by 1 , between optical properties evaluated with IDOSE or TOF.

the dosimetry software. Bare-end $600-\mu \mathrm{m}$ core diameter silica fibers (CeramOptec, Bonn, Germany) were inserted into the needles and extended $2 \mathrm{~mm}$ beyond the needle tip. A first monitoring sequence was run to establish optical properties and compute the first dose plan, then light delivery commenced. The light delivery was interrupted at intervals of 2, 4, 9, 14, 19, and 29 min for monitoring sequences. The dose plan was updated at each monitoring sequence. A constant light power of $150 \mathrm{~mW}$ was emitted from each fiber, and the illumination time for each fiber was varied to achieve a dose according to the dose plan.

The ability of online dosimetry to optimize the light dose plan was assessed using dose maps and dose-volume histograms $(\mathrm{DVH})$, and by reviewing the measurements from the monitoring sequences. Clinical assessments were made using MRI, prostate-specific antigen (PSA), and biopsy. Baseline MRI was done approximately two weeks before PDT using a protocol similar to that reported in Ref. 24. Follow-ups were then done at two weeks, eight weeks, six months, and

$$
\text { (a) }
$$

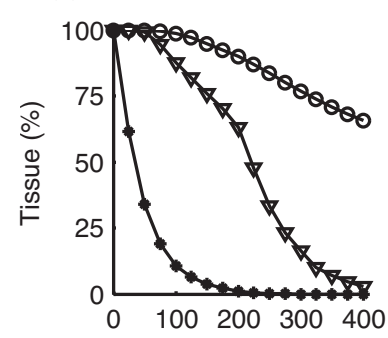

(c)

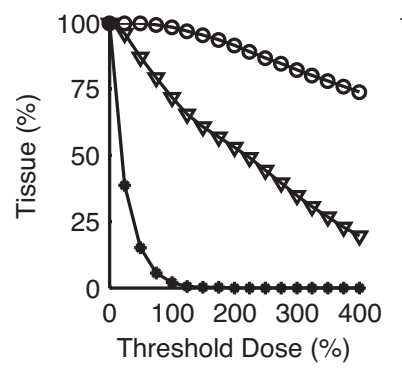

(b)

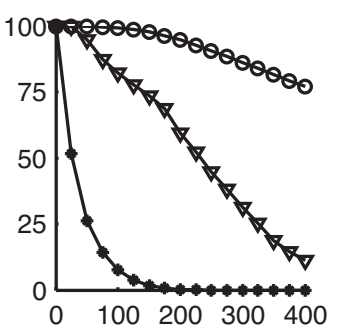

(d)

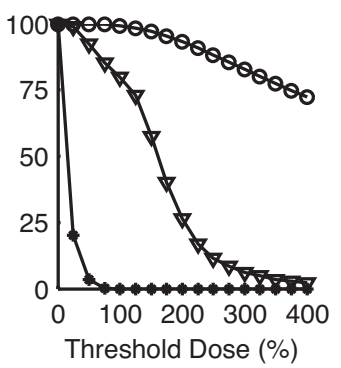

Fig. 2 Dose-volume-histogram for (a) patient 1, (b) patient 2, (c) patient 3, and (d) patient 4 . Three tissue types are included indicated by $(\bigcirc)$ prostate, $(\nabla)$ urethra, and $(\square)$ rectum.
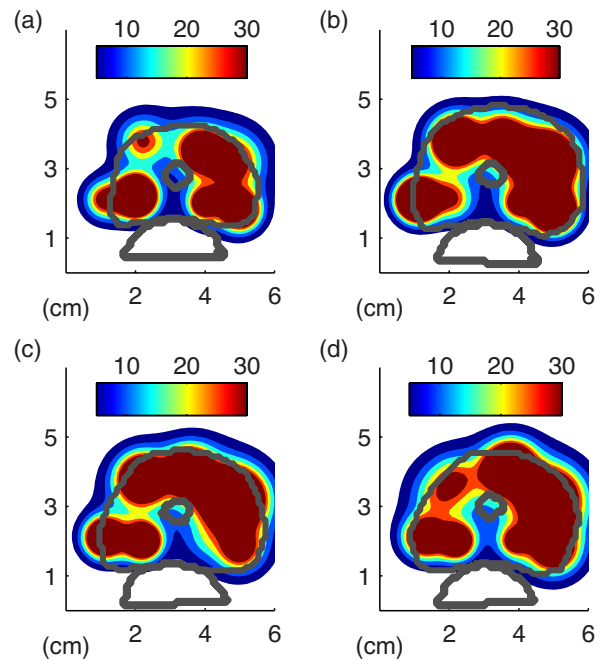

Fig. 3 Dose maps for patient 3, at the depths (a) 10, (b) 15, (c) 20, and (d) $25 \mathrm{~mm}$ along the craniocaudal axis. Color bar shows light fluence dose in joules per centimeters squared. (Color online only.)

12 months after PDT. PSA levels were checked regularly throughout the study. Twelve-core biopsy was done six months after PDT.

\section{Results}

\subsection{Tissue Phantom Experiments}

The evaluated effective attenuation coefficient for each tissue phantom is displayed in Fig. 1(a). It is seen that the steady state spatially resolved measurements performed within the IDOSE scheme underestimates the effective attenuation coefficient by $\sim 10 \%$ relative to the time-resolved evaluation. The evaluated optical properties were introduced into a real 3-D prostate organ model. This allowed the optimal treatment time and subsequently the light fluence dose to be calculated. By comparing the light dose calculated from spatially resolved measurements with the light dose calculated from timeresolved measurements, it was possible to determine the amount of treatment underestimation. Figure 1(b) shows the relative change, $\Delta \mathrm{TF}$ (in percent), of the treatment fraction due to underestimation of the effective attenuation coefficient. Treatment fraction represents the relative tissue volume of the total target volume that receives the threshold dose. Here, $\Delta \mathrm{TF}$ is defined by

$$
\Delta \mathrm{TF}=\frac{\mathrm{DVH}_{100 \%}^{\mathrm{TOF}}-\mathrm{DVH}_{100 \%}^{\mathrm{IDOSE}}}{\mathrm{DVH}_{100 \%}^{\mathrm{TOF}}} \times 100 .
$$

$\mathrm{DVH}_{100 \%}^{\mathrm{IDOSE}, \mathrm{TOF}}$ is the treatment fraction of the IDOSE of TOF scheme, respectively.

\subsection{Clinical Study}

Four patients, aged 70, 60, 56, and 67 years underwent Foscan-mediated PDT. The prostate volumes were 40 (36), 36 (46), 43 (46), and $30(40) \mathrm{cm}^{3}$, respectively, as determined by TRUS with an ellipsoid approximation (numbers in parentheses represent volumes evaluated by the voxel-based IDOSE 3-D tissue model). For all four patients, all 18 optical fibers 
Table 2 Dose metrics for patients $1-4$ compared to acceptance limits.

\begin{tabular}{|c|c|c|c|c|c|}
\hline \multirow[b]{2}{*}{$\begin{array}{l}\text { Tissue } \\
\text { Type }\end{array}$} & \multirow[b]{2}{*}{$\begin{array}{c}\text { Limit } \\
(\%)\end{array}$} & \multicolumn{4}{|c|}{ Patient } \\
\hline & & $\begin{array}{c}1 \\
(\%)\end{array}$ & $\begin{array}{c}2 \\
(\%)\end{array}$ & $\begin{array}{c}3 \\
(\%)\end{array}$ & $\begin{array}{c}4 \\
(\%)\end{array}$ \\
\hline Prostate & 95 & 98.7 & 99.2 & 98.3 & 99.1 \\
\hline Urethra & $<90$ & 87.4 & 82.3 & 71.6 & 79.7 \\
\hline Rectum & $<80$ & 10.7 & 7.7 & 2.0 & 0.0 \\
\hline Upper sph. ${ }^{a}$ & $<80$ & 22.0 & 54.9 & 8.4 & 0.0 \\
\hline Lower sph.a & $<50$ & 37.2 & 0.0 & 25.1 & 0.0 \\
\hline
\end{tabular}

were used to deliver therapeutic light and acquire measurements used for light fluence dose planning. In addition, two to four verification fibers were inserted at the capsule border and close to the rectal wall.

\subsubsection{Dosimetry}

The DVH, i.e., fraction of the tissue volume that receives a certain light fluence dose is presented in Figs. 2(a)-2(d) for all four patients. These DVHs are based on the calculated dose to the tissue after the treatment was finished. Prior to the start of the study, dose acceptance limits for the different tissue types were agreed on after consultation with the urologist. The acceptance limits are listed in Table 2 together with the dose metrics for all four patients. The dose metric values calculated for the sphincters differ significantly between the patients. This difference is due to smaller volumes of tissue compared to the other tissue types. Fig. 3 shows an example (patient 3 ) of calculated dose maps, which indicate the extent of the light dose distribution in the tissue. The dose maps represent calculated light fluence dose after the treatment, based on the 3-D geometry of the organs, the positions of the optical fibers, the measured optical properties at each fiber, and the known light energy delivered by each fiber. Figure 3 shows what parts of the different organs had received a light dose below or above the threshold dose, respectively. Figure 4 illustrates the predicted total light dose before and after each monitoring sequence during treatment progression as well as the final

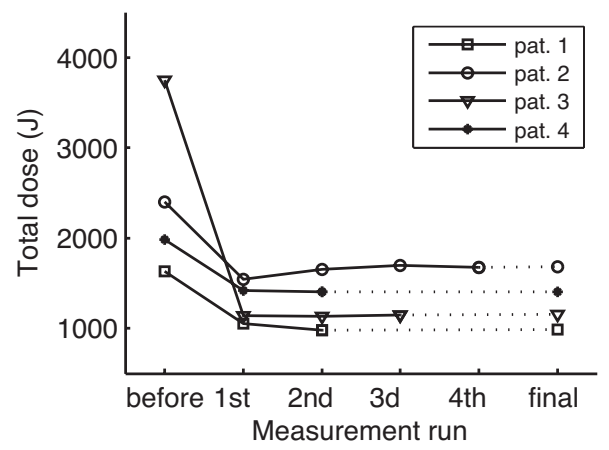

Fig. 4 The predicted total dose before and during treatment, as well as the final delivered dose for patient 1-4. dose delivered for all four patients. The predicted dose plan before measurements is calculated based on average optical properties taken from Ref. 26. Additional light delivery parameters are listed in Table 3.

\subsubsection{Optical properties}

The effective attenuation coefficient as calculated during treatment for each fiber is shown in Figs. 5(a)-5(d) for the four patients within the clinical trial. The error bars in Fig. 5 denote the standard deviation of all measurements performed during treatment, for each treatment fiber. The standard deviation seems to indicate that the optical properties did not vary markedly during treatment. The square markers denote evaluations that have an $r^{2}$-value above 0.5 .

\subsubsection{Clinical assessment}

There were no serious complications for any of the patients during the PDT treatment. The urinary catheters could be removed one to three days after treatment. The patients experienced urgency for about one week after the treatment. All patients, who previously had suffered from varying degree of voiding difficulty, experienced relief of urinary tract symptoms with a lowering of mean International Prostate Symptom Score. PSA values for all patients are shown in Fig. 6. PSA levels for all four patients were stabilized at a level below or at the level pre-PDT.

Table 3 Light delivery parameters for patients 1-4.

\begin{tabular}{lllll}
\hline Patient & 1 & 2 & 3 & 4 \\
\hline Max. time/fiber (min) & 12 & 29 & 15 & 14 \\
Average time/fiber (min) & 6 & 10 & 7 & 9 \\
Min. time/fiber (min) & 2 & 2 & 2 & 2 \\
Total light dose (J) & 986 & 1681 & 1155 & 1404 \\
Average dose/fiber $(\mathrm{J})$ & 55 & 93 & 64 & 78 \\
Dose per unit volume $\left(\mathrm{J} / \mathrm{cm}^{3}\right)$ & 27 & 37 & 25 & 35 \\
\hline
\end{tabular}




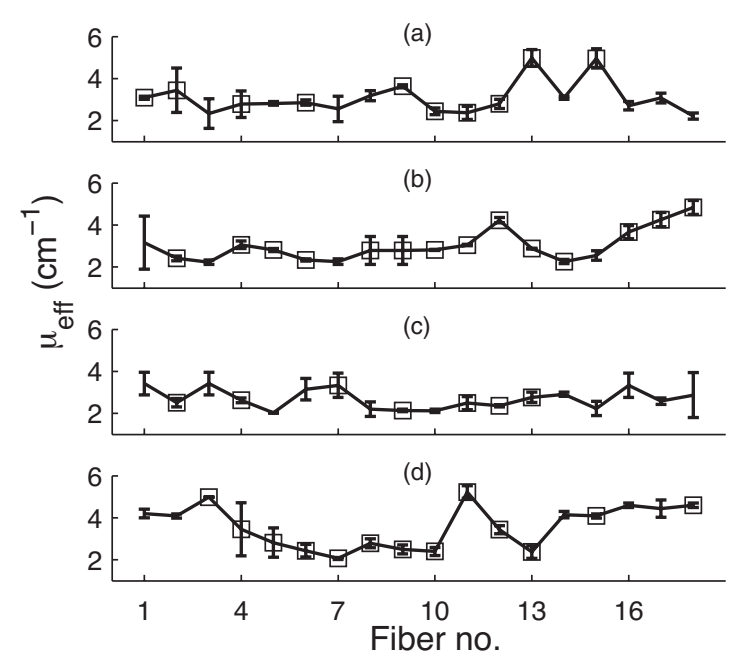

Fig. 5 The effective attenuation coefficient as a function of fiber number for (a) patient 1, (b) patient 2, (c) patient 3, and (d) patient 4 . The line indicates the mean of the effective attenuation coefficient for all monitoring sequences times where the error bars indicate \pm 1 standard deviation. The $\square$-marked values mark evaluations with $r^{2}$-value $\geqslant 0.5$.

MRI scans from patient 2 are shown in Fig. 7. Zones of decreased enhancement were visible in some cases two weeks post PDT, as illustrated in Fig. 7. No necrotic lesions were seen in the MRI images two months post PDT. The prostate volume was evaluated from MRI at baseline, two weeks and eight weeks post-PDT. A clear decrease (23-35\%) of the prostate volume was seen in all four patients, indicating a significant treatment response of the tissue. The volume reduction can be seen in the scans in Fig. 7. No tumors were visible on MRI scans in any of the patients.

Tissue biopsies taken at six months follow up revealed viable cancer cells in patients 1,2 , and 4 . Samples from patient 3 were benign, indicating either a successful treatment response or that the biopsy failed to sample viable cancer cells. Tumor viability was indicated by positive immunohistochemistry for proliferation, bcl-2 expression, and focal p53 upregulation. However, in all biopsies from the four patients, there were areas of secondary changes in the benign epithelium, such as vacuolisation, fibrosis, and hyaline degenera-

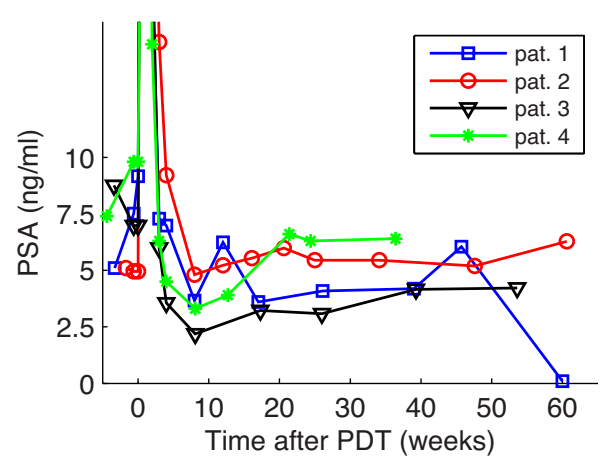

Fig. 6 PSA values from patients 1-4. Immediately after PDT, PSA rose to $40-78.1 \mathrm{ng} / \mathrm{ml}$. Patient 1 had a prostatectomy after 45 weeks. Patient 4 was withdrawn from the study at 35 weeks.

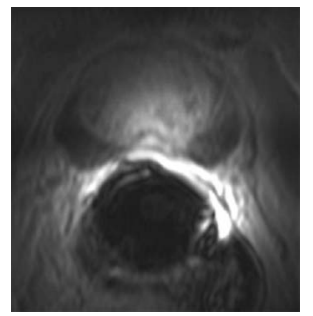

(a)

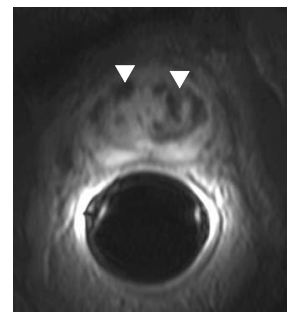

(b)
Fig. 7 (a) Baseline and (b) two weeks post-PDT contrast enhanced MRI scans from patient 2 . The dark structure below the prostate is the rectum. The arrows mark zones of reduced enhancement.

tion. These histological changes indicate a response to the treatment but seem to be focal and without involvement of confluent areas of necrosis.

\subsubsection{Adverse events}

No adverse events related to PDT in the target tissue or surrounding organs at risk have been reported in the clinical study. During the first PDT session, a technical problem caused a delay of $\sim 1 \mathrm{~h}$, where the patient suffered from a minor compartment syndrome, which may have been caused by the delay. Three of four patients have experienced skin photosensitivity, leading to skin burns due to too early sun exposure after photosensitizer administration. Patient 1 had skin burns on the injection arm due to accidental illumination by a surgical lamp, and similar burns appeared on the same arm, six months after PDT, due to sun exposure.

\section{Discussion}

\subsection{Tissue Phantom Experiments}

An underestimation of the effective attenuation will cause an alteration of the treatment volume. As seen in Fig. 1(b), the underestimation of the effective attenuation renders a lower treatment fraction; hence, the prostate tissue receives a lower light fluence dose, at worst $7 \%$ for phantom G. Despite a decrease of the treatment fraction the threshold dose is still reached in $90 \%$ of the prostate tissue for all phantoms. This outcome is in analogy with the discussion by Johansson et al., ${ }^{22}$ where it was concluded that the dosimetry algorithm adheres to a certain robustness against changes in the effective attenuation coefficient.

The effective attenuation coefficient is compared between IDOSE and a TOF spectroscopy system. It should be noted that after the validation experiments were performed, our group has reported on improvements on the evaluation scheme as well as a calibration method of time-resolved measurements. ${ }^{27,28}$ Herein it was discussed that the former rationale, adopted within this paper, slightly overestimated $\mu_{\mathrm{a}}$ and $\mu_{\mathrm{s}}^{\prime}$; hence, we believe that the accuracy might be better than shown in Fig. 1.

\subsection{Clinical Study}

\subsubsection{Dosimetry}

Figure 2 and Table 2 illustrate the success of the dosimetry module to meet the predefined acceptance limits. The acceptance limit of the urethra was intentionally set quite high be- 
cause the urethra was not deemed particularly sensitive, which is also verified by the limited time of catheterization. The dose maps for patient 3, in Fig. 3, indicate that the dosimetry algorithm is capable of shaping the light fluence dose, by adjusting the fiber-specific treatment times, to mainly target the prostate while limiting the dose to the urethra. It is especially noted that the rectum receives a very limited light dose.

The predicted dose for patient 3 (Fig. 4) is significantly higher than for the other patients before treatment. The predicted dose is primarily dependent on the prostate volume (which was largest for patient 3 according to the 3-D model). However, the shape of the prostate, its geometrical relation to surrounding organs at risk, and the optical fiber configuration also affect the predicted dose. These other factors explain why patient 2, who had a prostate of similar size, had a lower predicted dose before treatment.

\subsubsection{Optical properties}

The optical properties shown in Fig. 5 are within the range of previously reported values for human prostate. ${ }^{16,26,27}$ Svensson et al. ${ }^{26,27}$ reported on pronounced inter- and intrapatient variations of the optical properties. The effective attenuation coefficient assessed by IDOSE shows similar variations. These variations should be analysed with care because the time-resolved scheme employed by Svensson et al. ${ }^{26,27}$ probes the tissue between two optical fibers, whereas the IDOSE scheme probe a substantially larger portion of the prostate. The assumption made within the spatially resolved evaluation protocol is that the region around each treatment fiber is homogeneous. Hence, due to the evaluation model, we would expect a more homogeneous appearance of the effective attenuation coefficients because it averages $\mu_{\text {eff }}$ over a larger volume. Intrapatient variations due to abnormal prostate tissue features, such as calcifications, heterogeneous vascularization, and blood occlusions in front of fibers, will influence the measurements. Because the probed volume is large, such heterogeneites will appear as outliers in the measurements leading to poor linear fitting, which in turn can explain the low $r^{2}$-values in Fig. 5. As discussed by Johansson et al., ${ }^{22}$ and also seen in this paper, the dosimetry algorithm is still capable of delivering a predefined dose correctly when the effective attenuation coefficient is slightly altered. This means that the heterogeneous $\mu_{\text {eff }}$ has a limited effect on the dose calculations. On the other hand, potential calcifications and blood occlusions close to a source fiber can attenuate the treatment light, effectively decreasing the output power.

In order to assess how large effect potential output power perturbations will cause on the treatment fraction a simple first approximation is considered. The perturbation is mimicked by only imposing a multiplicative factor, within the interval $[0,1]$, on the output power when performing the dose calculations. The optical properties and final treatment times for the patients are considered, where the dose calculations are repeated 100 times for each patient. At each simulation, the perturbations are randomly sampled from a uniform distribution of the interval $[0,1]$. The treatment fraction for the prostate is seen together with the true treatment fraction, in Fig. 8, which indicates that, on average, the treatment fraction is diminished by $<20 \%$ due to occlusions in front of the fiber.

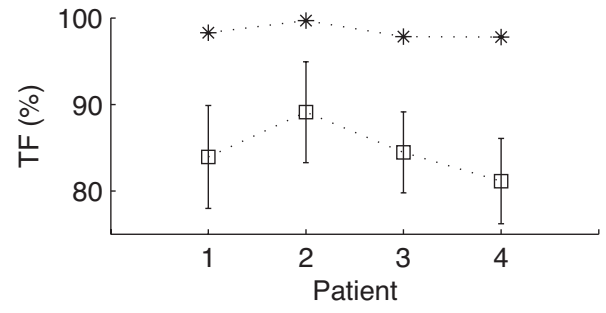

Fig. 8 Treatment fraction (TF) for the patient data where $(\star)$ denote true treatment fraction and $(\square)$ indicate treatment fraction when output power is perturbed. The error bars show \pm 1 standard deviation of 100 randomized samples of fiber-specific attenuation.

This analysis is based on the treatment fraction, which does not account for spatial variations within the target volume. This is significant because the local dose will be limited in situations when a fiber is subject to a high output power attenuation. In order to analyze the spatial dependencies on the fiber perturbations, a more rigorous approach is required.

\subsubsection{PSA}

The increase of PSA levels immediately after treatment is expected because leakage of PSA from the prostate organ is expected because the prostate tissue is destroyed by the PDT treatment. On the basis of experiences from radiotherapy, a decrease of PSA to levels below $0.5 \mathrm{ng} / \mathrm{ml}$ is then expected if most of the glandular and cancer tissue is destroyed. All four patients had stabilised PSA levels below the pretreatment values, but at a higher level than expected for total glandular tissue destruction. After radiotherapy, a definition of PSA relapse is a PSA nadir $+2 \mathrm{ng} / \mathrm{ml}$. On the basis of this definition, one patient had a relapse.

\subsubsection{MRI}

MRI cannot accurately verify anticancer effect of PDT because it is not possible to identify the cancer from the MR images, a fact that is due to the selection of patients. The MRI evaluation indicates that there is no harmful damage to the benign tissue. The volume reduction of the entire gland is clear and points to a treatment response in all parts of the prostate. The images are similar to the images from the Moore et al. study. ${ }^{24}$ The extent of the areas of reduced enhancement indicates undertreatment. In retrospect, the two weeks between PDT and MRI may have been longer than optimum to see a clear tissue response, because some healing and resolution of necrosis would have taken place.

\subsubsection{Biopsy}

All patients were initially diagnosed by examination of the tissue biopsies but at different time intervals before PDT. It was not possible to compare the biopsy results before and after PDT in any quantitative manner because the biopsy positions were different and the exact nature of the progression of the cancer between the first biopsy and PDT was unknown. The fact that three of four patients had residual viable cancer cells after PDT shows that the treatment goal, to ablate the entire prostate gland, was not met in these patients. 


\section{Conclusions}

This preliminary investigation, involving four patients, reports on the first clinical experiences using the SpectraCure P18 with IDOSE rationale for interstitial photodynamic therapy of prostate cancer with online feedback. The intention with the online dosimetry protocol is to deliver a tailored light fluence dose, exceeding a predefined threshold dose, to the whole prostate gland and adapt the dose plan in cases where the optical attenuation changes during therapy.

Preclinical validation of the instrument using tissue phantoms of varying optical properties showed that the system is capable of delivering a predefined dose within the acceptance limits to the target volume while sparing surrounding organs.

The conclusions form the clinical measurements indicate that the prostate tissue received under treatment. This is confirmed by higher PSA levels than expected as well as the presence of viable cancer cells in tissue biopsies, from three out of four patients, taken at the six-month follow-up. The MR images also support a below optimal light dose with only localized regions of decreased enhancement, indicating tumor response in those areas. Here, it should be noted that MRI was performed two weeks post-treatment, which may have allowed some resolution of necrosis to occur.

The explanation for the undertreatment is likely twofold. First, the predefined threshold dose was most likely set too low, and second, the output power from the treatment fibers seems to be attenuated by heterogeneities. Because this was the first clinical trial with completely automated real-time dosimetry, the threshold dose was chosen conservatively in order to avoid complications in surrounding organs at risk. In addition, the determination of the threshold dose relied on several assumptions about the optical properties and the necrosis radius from previous studies. Ideally, a more detailed threshold dose analysis should be performed where the necrosis and optical properties are assessed from the same individual.

The fiber-specific attenuation caused by heterogeneities, such as occlusions in front of the fibers, reduces the delivered dose to the target tissue. The simple analysis above shows that the treated fraction of the prostate volume could be $\sim 20 \%$ lower, although a more detailed analysis is required to truly assess the influence of this effect. As seen in the dose maps, in Fig. 3, in the absence of occlusions, the dose is well above the threshold dose within the prostate, a fact that is expected to somewhat alleviate the effect of fiber-specific attenuation.

To summarize, the first clinical experiences of the SpectraCure P18 with IDOSE system for treatment of prostate cancer show that the automated dosimetry system functions well and provides individually tailored dose plans online in the clinical treatment setting. Future work includes addressing the problem of attenuation by tissue heterogeneities at the optical fibers, finding the right threshold dose, and incorporating oxygen and photosensitizer data into the dosimetry rationale.

\section{Acknowledgments}

The authors greatly acknowledge Ann Johansson, Johan Stensson, Stephan Dymling, Sara Pålsson, and Tomas Johansson for their contributions to the development of instrumentation and dosimetry procedures. Additionally, the authors ac- knowledge Biolitec AG for providing the photosensitizer to this investigation. This work was supported by SpectraCure AB.

\section{References}

1. C. M. Moore, D. Pendse, and M. Emberton, "Photodynamic therapy for prostate cancer-a review of current status and future promise," Nat. Clin. Pract. Urol. 6(1), 18-30 (January 2009).

2. J. Trachtenberg, A. Bogaards, R. A. Weersink, M. A. Haider, A. Evans, S. A. McCluskey, A. Scherz, M. R. Gertner, C. Yue, S. Appu, A. Aprikian, J. Savard, B. C. Wilson, and M. Elhilali, "Vascular targeted photodynamic therapy with palladium-bacteriopheophorbide photosensitizer for recurrent prostate cancer following definitive radiation therapy: assessment of safety and treatment response," $J$. Urol. 178(5), 1974-1979; discussion, 1979 (November 2007).

3. J. Trachtenberg, R. A. Weersink, S. R. H. Davidson, M. A. Haider, A Bogaards, M. R. Gertner, A. Evans, A. Scherz, J. Savard, J. L. Chin, B. C. Wilson, and M. Elhilali, "Vascular-targeted photodynamic therapy (padoporfin, wst09) for recurrent prostate cancer after failure of external beam radiotherapy: a study of escalating light doses," BJU Int. 102(5), 556-562 (August 2008).

4. K. L. Du, R. Mick, T. Busch, T. C. Zhu, J. C. Finlay, G. Yu, A. G. Yodh, S. B. Malkowicz, D. Smith, R. Whittington, D. Stripp, and S. M. Hahn, "Preliminary results of interstitial motexafin lutetiummediated pdt for prostate cancer," Lasers Surg. Med. 38(5), 427-434 (2006).

5. K. Verigos, D. C. H. Stripp, R. Mick, T. C. Zhu, R. Whittington, D. Smith, A. Dimofte, J. Finlay, T. M. Busch, Z. A. Tochner, S. B. Malkowicz, E. Glatstein, and S. M. Hahn, "Updated results of a phase I trial of motexafin lutetium-mediated interstitial photodynamic therapy in patients with locally recurrent prostate cancer," J. Environ. Pathol. Toxicol. Oncol. 25(1-2), 373-387 (2006).

6. R. A. Weersink, A. Bogaards, M. Gertner, S. R. H. Davidson, K. Zhang, G. Netchev, J. Trachtenberg, and B. C. Wilson, "Techniques for delivery and monitoring of tookad (wst09)-mediated photodynamic therapy of the prostate: clinical experience and practicalities," J. Photochem. Photobiol., B 79(3), 211-222 (2005).

7. B. C. Wilson and M. S. Patterson, "The physics, biophysics and technology of photodynamic therapy," Phys. Med. Biol. 53(9), R61R109 (May 7, 2008).

8. X. D. Zhou, B. W. Pogue, B. Chen, E. Demidenko, R. Joshi, J. Hoopes, and T. Hasan, "Pretreatment photosensitizer dosimetry reduces variation in tumor response," Int. J. Radiat. Oncol., Biol., Phys. 64(4), 1211-1220 (2006).

9. J. C. Finlay, T. C. Zhu, A. Dimofte, D. Stripp, S. B. Malkowcz, T. M. Busch, and S. M. Hahn, "Interstitial fluorescence spectroscopy in the human prostate during motexafin lutetium-mediated photodynamic therapy," Photochem. Photobiol. 82(5), 1270-1278 (2006).

10. A. Dimofte, T. C. Zhu, S. M. Hahn, and R. A. Lustig, "In vivo light dosimetry for motexafin lutetium-mediated pdt of breast cancer," Lasers Surg. Med. 31, 305-312 (2002).

11. J. Li and T. C. Zhu, "Determination of in vivo light fluence distribution in a heterogeneous prostate during photodynamic therapy," Phys. Med. Biol. 53(8), 2103-2114 (April 21, 2008).

12. T. M. Busch, E. P. Wileyto, M. J. Emanuele, F. Del Piero, L. Marconato, E. Glatstein, and C. J. Koch, "Photodynamic therapy creates fluence rate-dependent gradients in the intratumoral spatial distribution of oxygen," Cancer Res. 62(24), 7273-7279 (2002).

13. T. M. Busch, "Local physiological changes during photodynamic therapy," Lasers Surg. Med. 38(5), 494-499 (June 2006).

14. H. W. Wang, T. C. Zhu, M. E. Putt, M. Solonenko, J. Metz, A. Dimofte, J. Miles, D. L. Fraker, E. Glatstein, S. M. Hahn, and A. G. Yodh, "Broadband reflectance measurements of light penetration, blood oxygenation, hemoglobin concentration, and drug concentration in human intraperitoneal tissues before and after photodynamic therapy," J. Biomed. Opt. 10, 014004 (2005).

15. S. R. Davidson, R. A. Weersink, M. A. Haider, M. R. Gertner, A Bogaards, D. Giewercer, A. Scherz, M. D. Sherar, M. Elhilali, J. L. Chin, et al., "Treatment planning and dose analysis for interstitial photodynamic therapy of prostate cancer," Phys. Med. Biol. 54(8), 2293-2313 (2009). 
16. T. C. Zhu, A. Dimofte, J. C. Finlay, D. Stripp, T. Busch, J. Miles, R. Whittington, S. B. Malkowicz, Z. Tochner, E. Glatstein, and S. M Hahn, "Optical properties of human prostate at $732 \mathrm{~nm}$ measured in vivo during motexafin lutetium-mediated photodynamic therapy," Photochem. Photobiol. 81(1), 96-105 (2005).

17. G. Q. Yu, T. Durduran, C. Zhou, T. C. Zhu, J. C. Finlay, T. M. Busch, S. B. Malkowicz, S. M. Hahn, and A. G. Yodh, "Real-time in situ monitoring of human prostate photodynamic therapy with diffuse light," Photochem. Photobiol. 82(5), 1279-1284 (2006).

18. M. D. Altschuler, T. C. Zhu, J. Li, and S. M. Hahn, "Optimized interstitial pdt prostate treatment planning with the cimmino feasibility algorithm," Med. Phys. 32(12), 3524-3536 (2005).

19. J. Li, M. D. Altschuler, S. M. Hahn, and T. C. Zhu, "Optimization of light source parameters in the photodynamic therapy of heterogeneous prostate," Phys. Med. Biol. 53(15), 4107-4121 (2008).

20. T. Johansson, M. Soto Thompson, M. Stenberg, K. Claes af, S Andersson-Engels, S. Svanberg, and K. Svanberg, "Feasibility study of a system for combined light dosimetry and interstitial photodynamic treatment of massive tumors," Appl. Opt. 41(7), 1462-1468 (March 2002).

21. M. Soto-Thompson, A. Johansson, T. Johansson, S. AnderssonEngels, S. Svanberg, N. Bendsoe, and K. Svanberg, "Clinical system for interstitial photodynamic therapy with combined on-line dosimetry measurements," Appl. Opt. 44(19), 4023-4031 (July 2005).
22. A. Johansson, J. Axelsson, S. Andersson-Engels, and J. Swartling, "Realtime light dosimetry software tools for interstitial photodynamic therapy of the human prostate," Med. Phys. 34(11), 4309-4321 (November 2007).

23. A. Johansson, J. Axelsson, J. Swartling, T. Johansson, S. Palsson, J. Stensson, M. Einarsdottir, K. Svanberg, N. Bendsoe, K. M. Kalkner, S. Nilsson, S. Svanberg, and S. Andersson-Engels, "Interstitial photodynamic therapy for primary prostate cancer incorporating realtime treatment dosimetry," Proc. SPIE 6427, 642700 (2007).

24. C. M. Moore, T. R. Nathan, W. R. Lees, C. A. Mosse, A. Freeman, M. Emberton, and S. G. Bown, "Photodynamic therapy using meso tetra hydroxy phenyl chlorin (mthpc) in early prostate cancer," Lasers Surg. Med. 38(5), 356-363 (2006).

25. C. M. Moore (personal communication).

26. T. Svensson, M. Einarsdottir, K. Svanberg, and S. Andersson-Engels, "In vivo optical characterization of human prostate tissue using nearinfrared time-resolved spectroscopy," J. Biomed. Opt. 12(1), 014022 (2007).

27. T. Svensson, E. Alerstam, M. Einarsdottir, K. Svanberg, and S Andersson-Engels, "Towards accurate in vivo spectroscopy of the human prostate," J. Biophoton. 1(3), 200-203 (2008).

28. E. Alerstam, S. Andersson-Engels, and T. Svensson, "Improved accuracy in time-resolved diffuse reflectance spectroscopy," Opt. Express 16(14), 10440-10454 (July 2008). 